\title{
Development of an electrical impedance based spirometer
}

\author{
Md. Anas Ali, M Abdul Kadir and K Siddique-e Rabbani \\ Department of Biomedical Physics \& Technology, University of Dhaka, Dhaka 1000, Bangladesh \\ E-mail: anasalilimon@gmail.com,kadir@du.ac.bd,rabbani@du.ac.bd
}

\begin{abstract}
Spirometry, or measurement of respiration conventionally involves measurement of the time variation of volume or flow of air breathed in or out through the mouth using an air-flow sensor. Electrical impedance of thorax changes during breathing and conceptually this modality should be applicable in spirometry, but no attempts have been made so far. This paper describes the development of an electrical impedance based spirometer placing electrodes on four limbs so that the whole lung volume may contribute to the measurement. An impedance based measurement system (IBS) consisting of a constant current drive at 11 $\mathrm{kHz}$ and necessary voltage measurement circuitry was designed and developed for this purpose. A portable oscilloscope (PicoScope 2208A) was used for acquisition of the measured voltage signal to a PC and an algorithm in Matlab software was used to demodulate the time varying impedance from a carrier signal. The variation of impedance with time during forced expiration as measured by the implemented impedance measurement system was compared with that obtained using a standard bellows type commercial spirometer (Vitalograph) on eight volunteers, between 22 and 32 years of age. The subjects included smokers, nonsmokers, and asthma patients. Repeatability of the measurements were satisfactory. The correlation coefficient between the spirogram obtained using the IBS and that obtained using standard spirometer varied from 0.81 to 0.99 indicating preliminary success of the proposed technique. However, a possible source of error is the bending of the body during forced expiration which, if prevented, may give a better outcome.
\end{abstract}

Keywords: Electrical Impedance Based Spirometer, Spirometry, Spirometer, Bioimpedance, Electrical Impedance

\section{INTRODUCTION}

Spirometer is a device used to assess the functionality of human lungs (Enright et al., 1991). A spirometer usually measures the volume and flow of air inhaled or exhaled by a person as a function of time (Vijayasekaran et al., 2003). In spirometry, a subject holds a tube tightly between the lips, closes his or her
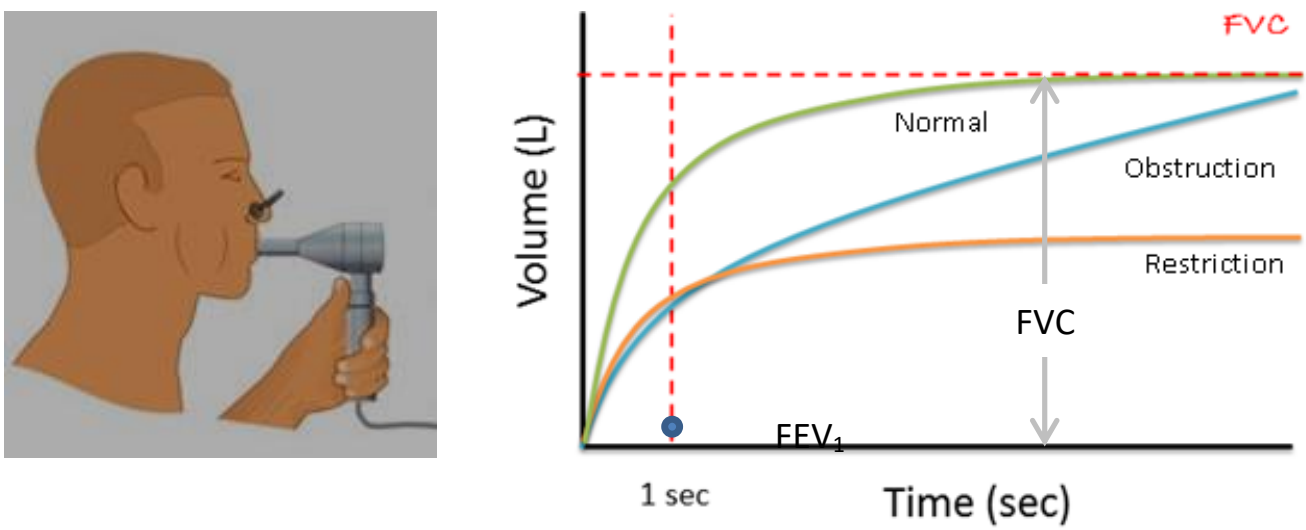

Fig.1: Spirometric measurement procedure and typical measured parameters for forced expiration which indicates obstructive and restrictive conditions of lungs. 
nostrils using a clip, and then breathes in or out of this tube which is attached to a flow or a volume sensor as shown in the left side of Fig.1. An often measured procedure involves breathing in as much as possible, holding breath for a moment, and then breathing out forcefully as quickly as possible, to empty the lungs as much as possible. In this measurement, the volume of air breathed out forcefully is plotted against time as shown in the right side of Fig.1. The maximum volume expired after maximum inhalation is called Forced Vital Capacity (FVC). The volume exhaled at a specific time such as $1 \mathrm{sec}$ is called Forced Expiratory Volume at one second $\left(\mathrm{FEV}_{1}\right)$, also shown in Fig.1. Both FVC and $\mathrm{FEV}_{1}$ are useful in detecting lungs disorders that result in obstruction and restriction which typically occur in asthma, COPD and pulmonary fibrosis. COPD stands for Chronic Obstructive Pulmonary Disease that is characterized by a significantly reduced ratio of $\mathrm{FEV}_{1} / \mathrm{FVC}$ (Barnes and Fromer, 2011). However, a reduction in FVC may also be present. Asthma is also an obstructive airway disease (OAD) which is indicated by reduced ratio of $\mathrm{FEV}_{1} / \mathrm{FVC}$. In restrictive pulmonary diseases (Saxena, 2015) like pulmonary fibrosis, FVC is reduced. Fig. 1 shows typical volume-time plots for lungs under normal condition, obstruction and restriction. There are other modes of spirometry as well, but this paper concentrates on the volume-time plot as described above.

Different types of sensors are used in spirometers, sensing either flow or volume, and the results can be interchanged through mathematical manipulation. Sensors employing a turbine or a wire mesh are most popular. However, performance of turbine type flow sensors are limited by friction and inertia while the mesh type has the problem of water droplets accumulation in the mesh when a person blows into it (Schermer et al., 2012). Again, in all of the traditional devices, the tube into which a person blows has a certain diameter which, however large, may obstruct the flow to a certain extent when a person blows into it. Therefore, the flow that is actually obtained is not fully unobstructed in the true sense. Importantly, many subjects including children are not capable in exhaling through the pipe of a spirometer properly (Jat, 2013).

To overcome these limitations, the present work was taken to develop an electrical impedance based spirometer (IBS). The dielectric properties of lung tissue changes as a function of air content within the lungs (Nopp et al., 1993). The electrical conductivity of inflated and deflated lung tissue was reported to be $0.093 \mathrm{~S} / \mathrm{m}$ and $0.243 \mathrm{~S} / \mathrm{m}$ respectively at $10 \mathrm{kHz}$ (Andreuccetti $\mathrm{D}, 1997$ ). This suggests that electrical conductivity of lung tissue during expiration is more than two fold higher than that during inspiration. Electrical bioimpedance is the opposition to a flow of current through a biological tissue that depends on the dielectric properties of that particular tissue (Martinsen and Grimnes, 2011). Kubicek at al. reported a linear relationship between change in transthoracic impedance and lung air volume (Kubicek et al., 1964). They suggested that respiratory volumes might be obtained from transthoracic impedance changes during respiration. Kadir et al. also reported a linear relationship between electrical impedance of a localized region in chest measured using skin electrodes to the volume of air breathed out (Kadir et al., 2009, Kadir et al., 2015). However, no attempts have been made so far to use electrical impedance techniques in spirometry.

This paper describes an electrical impedance based spirometer requiring no turbine, no mesh, not even any tube to blow through so that there is no obstruction due to the device itself. The present work can be divided into two parts. The first part involves development of necessary instrumentation and software for measurement and recording the change in electrical impedance during breathing. In the second part, preliminary measurements of lung air volumes were performed with the developed IBS system on human subjects and compared with that obtained from a standard spirometer. 


\section{METHODS AND MATERIALS}

Electrical impedance of the thoracic region can be divided into two components: a relatively steady value $Z$ contributed by different types of tissues in the thorax, and a varying value $\Delta Z$ due to ventilation in lungs (Gupta, 2011). Therefore, the total impedance is given by,

$$
\mathrm{Z}_{\mathrm{T}}=\mathrm{Z}+\Delta \mathrm{Z}
$$

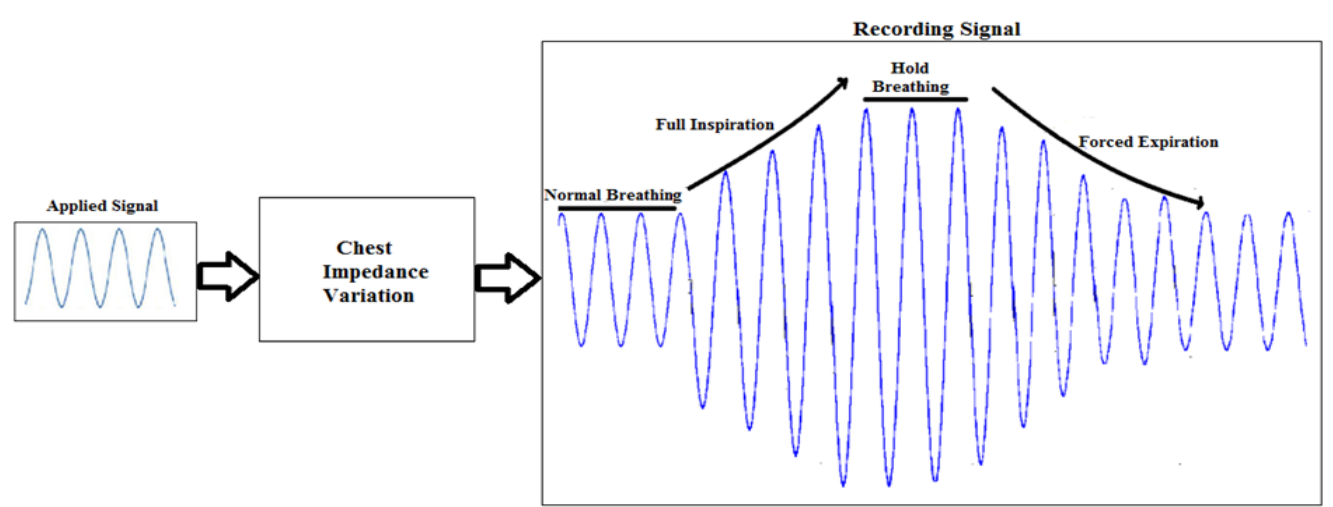

Fig.2: Expected variation of impedance (right side) of the thoracic region on different breathing maneuvers for an input of constant amplitude sinusoidal signal (left).

In bioimpedance techniques, usually an alternative current of constant amplitude is injected through a pair of drive electrodes into the thorax region and the corresponding potential difference across another pair of sense electrodes is measured known as tetra-polar impedance measurement (TPIM). Since injected current is constant, the measured potential is proportional to the impedance of the thorax termed as transfer impedance (Grimnes and Martinsen, 2008). The measured transfer impedance increases during inspiration and decreases during expiration, but remains fixed at breathhold. Fig. 2 shows the expected variation impedance of thorax for different breathing maneuvers of a subject. Through a differential measurement between inhalation and exhalation, $\Delta \mathrm{Z}$ can be obtained since the fixed $\mathrm{Z}$ part cancels out on subtraction. 


\subsection{Instrumentation}

The block diagram of the developed thoracic impedance measurement system based on TPIM is shown in Fig.3. It essentially consists of two major blocks, signal injection circuitry and bio-potential measurement circuitry. The signal injection segment is essentially an AC source with constant amplitude. A commercial signal generator (GWINSTEK SFG-2004) was used to provide a sinusoidal signal of constant voltage amplitude at $10 \mathrm{kHz}$. This sinusoidal signal was then fed to an improved Howland current source. The output of the current source is a constant current of amplitude $1 \mathrm{~mA}(\mathrm{p}-\mathrm{p})$ at $10 \mathrm{kHz}$ which is considered safe for human (AAMI, 1985). In order to isolate the mains electrical supply from the patient connected parts, an analog optical isolation circuit was used in between the signal generator and the Howland current source.

The first stage of the voltage measurement circuit is a differential amplifier with high common mode rejection ratio. For noise cancellation, a Butterworth band pass filter circuit was used since Butterworth type filter optimizes between number of components and other characteristics like step response and component tolerance sensitivity. The center frequency of the pass band filter was $10 \mathrm{kHz}$ and with a band width of $1 \mathrm{kHz}(9.5 \mathrm{kHz}$ to $10.5 \mathrm{kHz})$ at an attenuation of -3dB. Finally the signal is again amplified before it was fed to a digital data acquisition system. The overall gain of the potential measurement circuitry was 1200.

For the digital data acquisition, a PC based portable oscilloscope (PicoScope, model: 2208A, Software version: 6.11.12) was used. The amplified voltage signal was acquired with sampling rate of $200 \mathrm{~K}$ samples per second, which gave 20 data points per cycle, enough to represent the sinusoidal signal giving a correct representation of the amplitude, which is the parameter to be measured in the present work. The PicoScope data acquisition module has two input channels and is connected to a PC through USB port and obtains power from the PC. One of these channels was used for acquiring the voltage value from the TPIM system while the other was used to acquire data from a commercial spirometer as described later.

A wedge bellows type commercial spirometer 'Vitalograph' (Drew and Hughes, 1969) was used for standard measurements of lung volumes. The Vitalograph usually gives spirometric values in a paper graph using a mechanical system. However, fixing a linear potentiometer with the axle of the bellows, the device was modified to give an electrical output. A DC constant voltage was applied to the two outer ends of the potentiometer so that the voltage between the central terminal of the potentiometer and any of the ends give a measure of the angle of rotation. This output voltage was then calibrated with

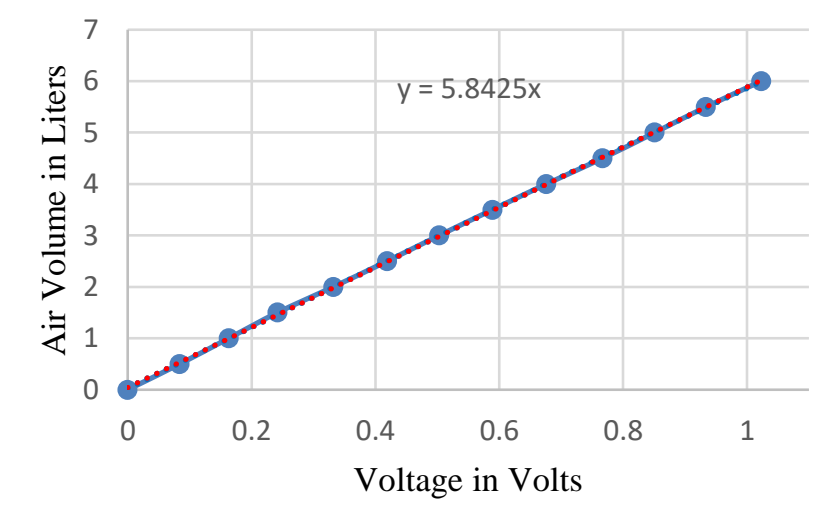

Fig.4: Calibration curve of the modified 'Vitalograph' in this work for obtaining air volume from voltage values. respect to the volume values shown on the plotted graph. The calibration curve is shown in Fig.4.

\subsection{Lung volume measurements}

Lung air volume measurements were performed on 8 human subjects, all adults, aged between 22 and 32 
years. Four of them were non-smokers (P1, P4, P5, P6) and remaining 4 were smokers (P2, P3 P7, P8). The subjects had no known respiratory disorder except one (P3) who had diagnosed mild obstructive lung disease (asthma). The subjects were well trained in the manoeuvers needed for the measurements beforehand.

The current drive electrodes of the TPIM system were attached to the right hand and right leg while the potential measuring electrodes were attached on left hand and left leg respectively, as shown in Fig.3. Standard ECG clip electrodes were used with electrode gel between skin and the metal electrode. During the test the subject sat on a chair in an erect position and continued normal breathing. Then the subject performed a full inhalation, held breath for a few seconds, and then exhaled forcefully into the flow tube of the Vitalograph as much as possible. This gave the volume of exhaled air through the electrical sensing system of Vitalograph as described before. Simultaneously, the electrical impedance was also measured using the IBS system. The PicoScope device acquired voltage values from both the IBS system and the Vitalograph into a computer allowing air volume measurements using both the systems simultaneously as shown in Fig.5.

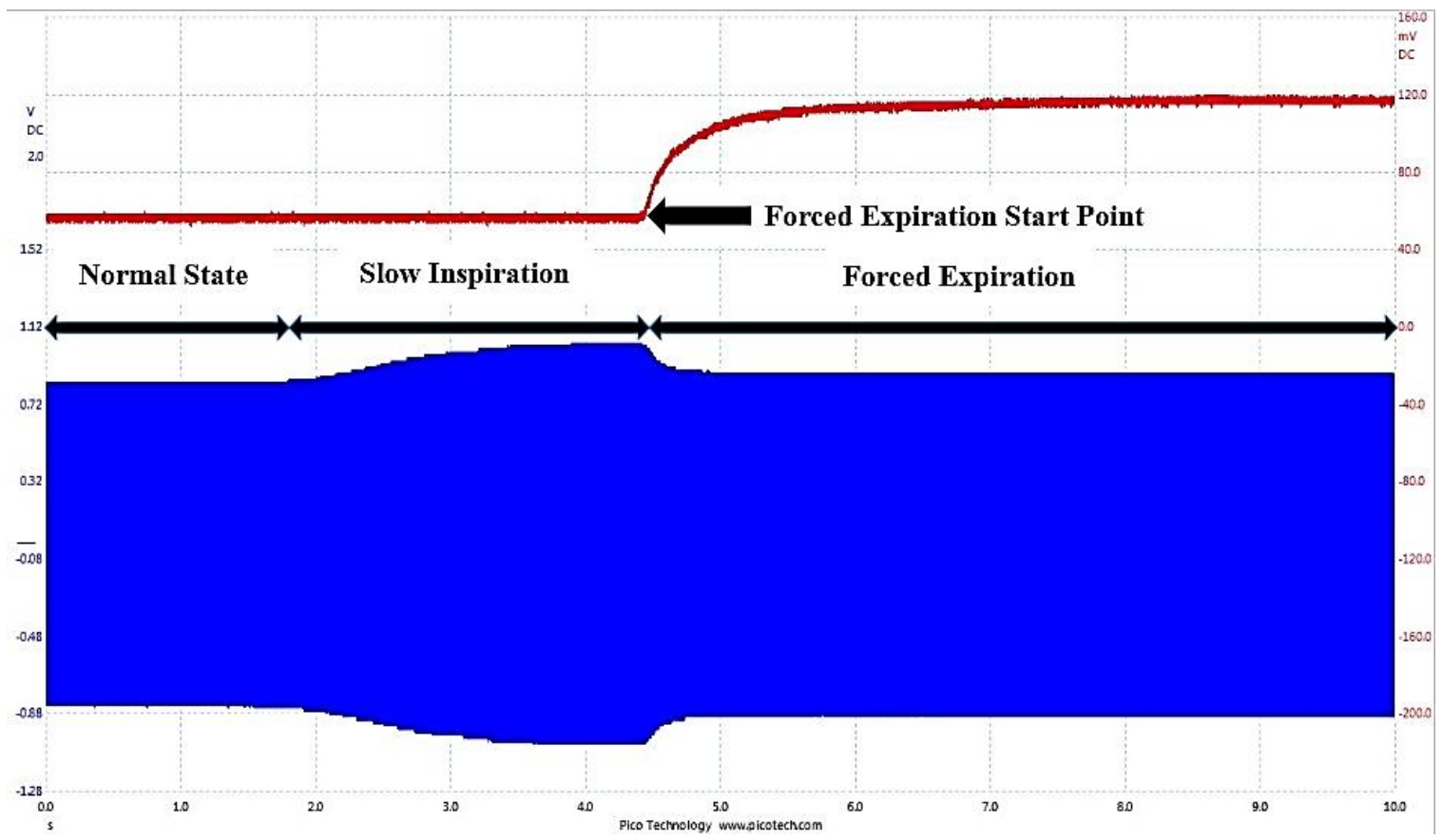

Fig.5: Data recorded by PicoScope, the red curve represents voltage changes of the Vitalograph against time while the blue shaded area represents impedance changes measured by IBS system during respiration as the peak to peak variation of the $10 \mathrm{kHz}$ signal. The vertical axis is labeled in voltage, left side (in $\mathrm{V}$ ) for IBS and right side (in $\mathrm{mV}$ ) for Vitalograph.

In Fig.5, the dark red curve represents the voltage output of the Vitalograph and blue band represents the output of the IBS system. Since the time scale of the graph has been set at seconds, the recorded $10 \mathrm{KHz}$ sinusoidal signal of the IBS becomes compressed and appears as a band with varying amplitudes. The amplitude change on either side has the relevant information of lungs impedance change. However, during the time that the subject inspires air, the amplitude of the IBS also increases which does not appear in the Vitalograph system because air is inspired from outside the Vitalograph tube and it is not designed to 
measure inspired volume. The comparison has to be made only for the segment following the start of the forced expiration, as indicated in Fig.5. The horizontal axis represents time (in sec) which is the same for both measurements. The left vertical axis is labeled in Volts for IBS while the right vertical axis is labeled in $\mathrm{mV}$ for Vitalograph. It is to be noted from Fig.4 that the voltage output of Vitalograph will be proportional to the volume of air breathed out.

The impedance variation due to respiration measured by the IBS system was available as a modulated $10 \mathrm{kHz}$ signal as shown in Fig 5. It is necessary to demodulate the peak values to get the desired IBS signal, which would be proportional to the electrical impedance. An algorithm was developed to demodulate the $10 \mathrm{kHz}$ signal to obtain the time variation of impedance due to respiration, as shown in the flow diagram in Fig.6. This was implemented in MATLAB software. From the IBS data, firstly, the upper peak points of the sinusoidal signals with respect to zero level were detected. A moving average of these peak points was performed to smooth out the data. As can be seen from Fig.5, the direction of change of the top part of the IBS band is just the opposite to that of the Vitalograph. This is because as the air is blown out of the lungs, the electrical impedance decreases while the volume recorded by the Vitalograph increases. Therefore, these data from the IBS had to be inverted to

$\longrightarrow$ Standard Spirogram $\longrightarrow$ Impedance Spirogram

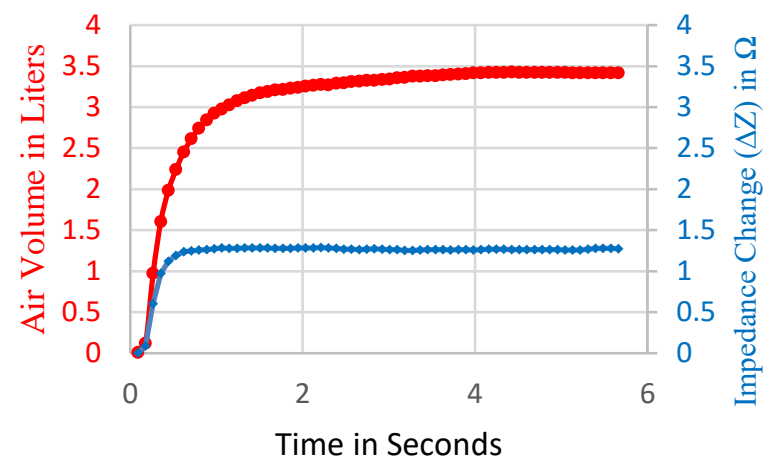

Fig.7: Spirogram of the recorded signal shown in Fig.4. The red curve shows the time variation of expired air volume recorded by the Vitalograph, considered as standard. The blue curve shows the impedance change with time, measured using the IBS.

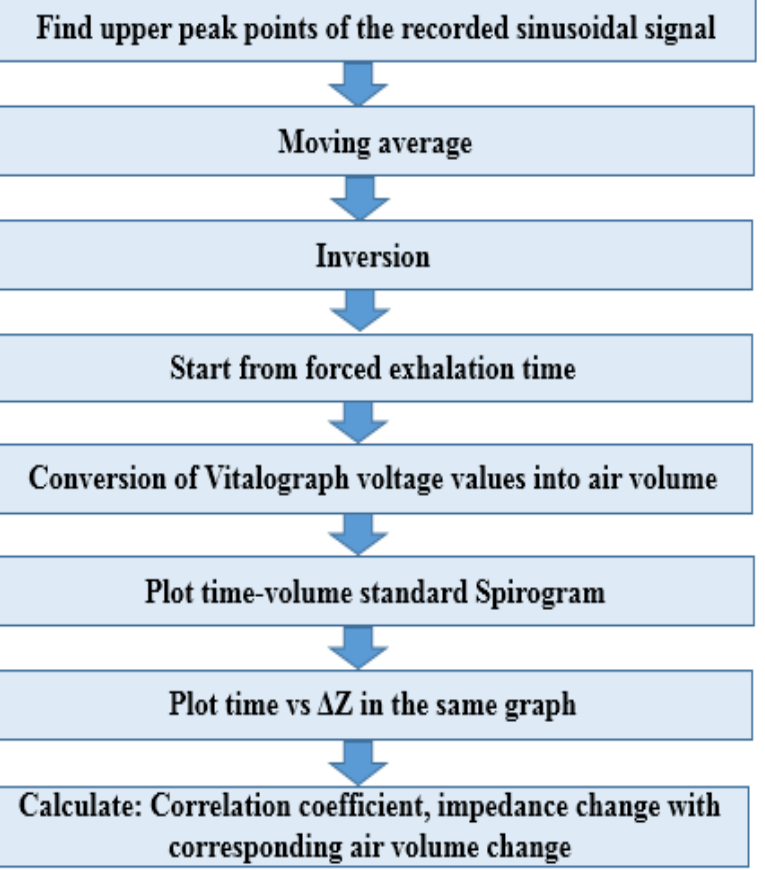

Fig.6: Flow diagram of the developed algorithm for demodulation of the time variation of impedance implemented in MATLAB software. get a plot of impedance against time. The software also plotted the volume output of the Vitalograph and simultaneous recording of both plots are shown in Fig.7.

Both the curves obtained from the Vitalograph and the IBS can be compared visually in the same direction of the vertical axis. Both the curves were to be started from the instant of time that the subject started to exhale forcefully. This point was chosen manually from the PicoScope display on the PC. The Vitalograph output was shown in terms of air volume exhaled using the calibration curve shown in Fig.4 while the IBS output was shown in terms of the measured transferred impedance change, expressed in ohms. To obtain these numerical values, the recorded voltage values of Vitalograph were converted into air 
volumes using equation 2 which uses the value of slope (=5.84) of the calibration curve given in Fig.4. Therefore,

$$
\text { Volume }=5.84 \times \text { Vitalograph voltage } \ldots \text { (2) }
$$

The variation of transfer impedance $(\Delta \mathrm{Z})$ was obtained dividing the change in voltage output $(\Delta \mathrm{V})$ by the constant current $(=1 \mathrm{~mA})$ injected in the IBS, as given in equation 3 .

$$
\text { Impedance Variation }(\Delta V)=\frac{\text { Potential change }(\Delta \mathrm{V})}{\text { Injected current } \times \text { Gain }}
$$

\section{RESULTS AND OBSERVATIONS}

The current driven by the current drive circuitry through variable load resistors was plotted in Fig.8. Although a little fluctuation of current occurs with increasing load but it is negligible $(<1 \%)$ up to $8 \mathrm{k} \Omega$ and current almost remains fixed at $1 \mathrm{~mA}(\mathrm{p}-\mathrm{p})$. The sum of contact impedance, tissue impedance and change in impedance due to respiration is typically much less than $8 \mathrm{k} \Omega$ at $10 \mathrm{kHz}$. Hence, it can be said that the performance of the current source is acceptable for the application envisaged.

Repeatability is an important condition for any

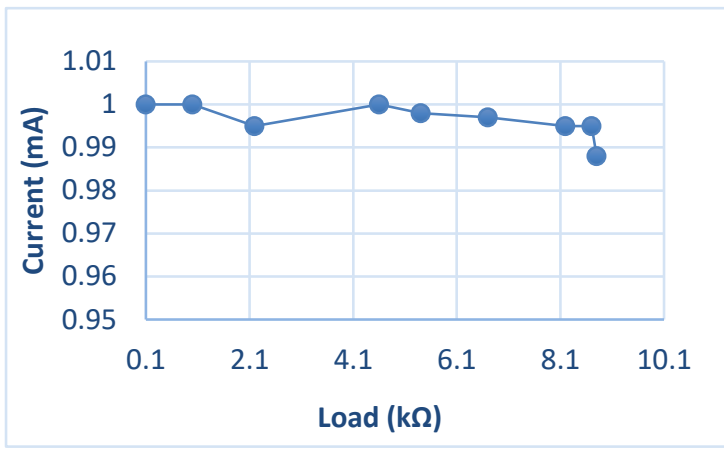

Fig.8: Performance of current drive circuit, variation current with load resistors. measurement. Three repeated measurements were taken for one subject (P4), using both the Vitalograph and the developed IBS. The variation of air volume measure by Vitalograph and the variation of impedance measured by IBS are shown in Fig.9 with different coloured curves. It can be seen that both the measurements are fairly reproducible.

To match the impedance variation with the corresponding air volume variation shown in Fig.9, a conversion

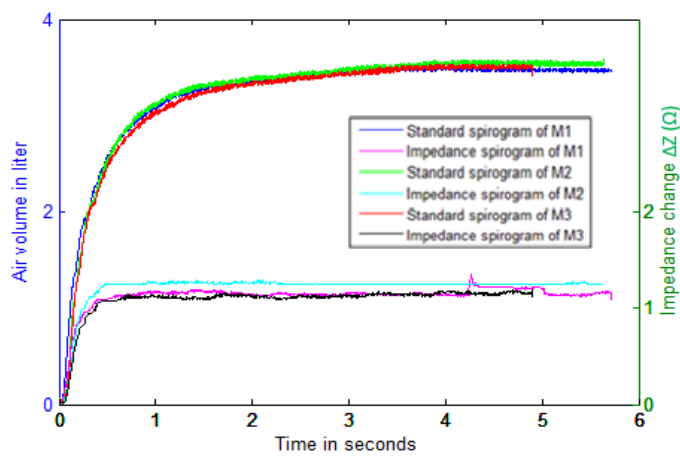

Fig.9: Measurement repeatability of spirogram obtained from both IBS and Vitalograph. factor (CF) was introduced that would convert the maximum change in impedance value to the total air volume exhaled (FVC). It was found that for subject P4 a factor of 2.70 litres/ohms gave a reasonable match between the measurements taken using the Vitalograph and the IBS (Fig.10). Although it was matched for the FVC (later part of the plot), there was observable difference during the initial period, near the sharp rising part of the spirogram. Besides, the factor was not the same for the different subjects as shown in Table 1 and Fig.11. The average of the conversion factor was found to be 2.74 with a standard deviation of 0.45 , both in liters/ohms. Taking this mean as the conversion factor for all the subjects, the estimated

FVC values based on IBS were also shown in Table 1, which also shows the individual errors considering the Vitalograph measurements as standards. Except one, P6, all the other subjects have errors under about $13 \%$. It needs to be seen how this dispersion can be minimized through improvement and standardization of measurement procedures. Of course, there may be some dependence on age groups, gender and body 
frame size. Compensation for these factors may reduce the dispersion giving an improved common factor for the conversion.

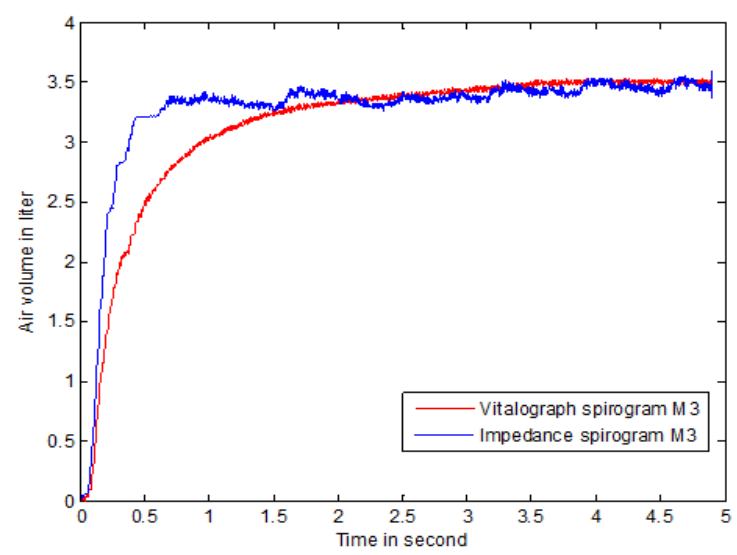

Fig.10: Volume-Time graphs of a subject (P4) measured using both Vitalograph (red line) and IBS (blue line). A factor of 2.7 liters/ohms was used for the latter.

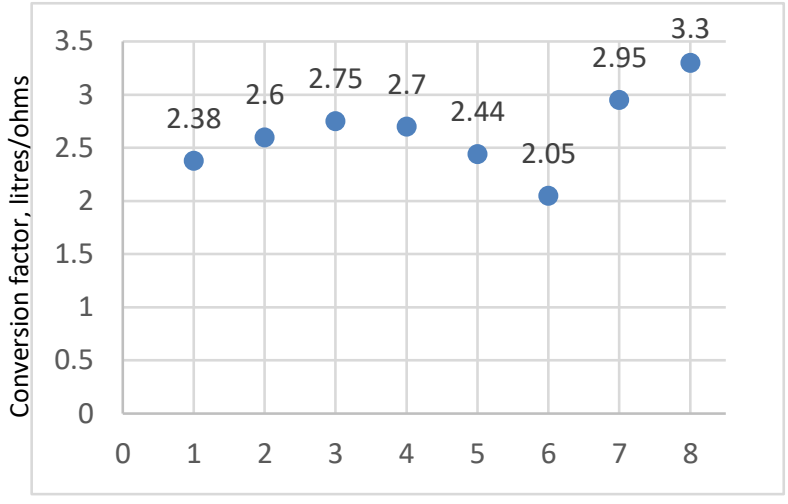

Fig. 11: Factors for conversion of impedance change to FVC best suited to each of the 8 subjects.

Fig.12 shows time variation of lung air volume measured using IBS and Vitalograph for four subjects. Three of them were smokers (P2, P7, P8) while one (P3) had diagnosed asthmatic condition. A large difference can be observed for the subject with asthma (P3) where the IBS did not match the reduction in volume in the early period of exhalation. However, from the pattern of the straight line for the final segment,
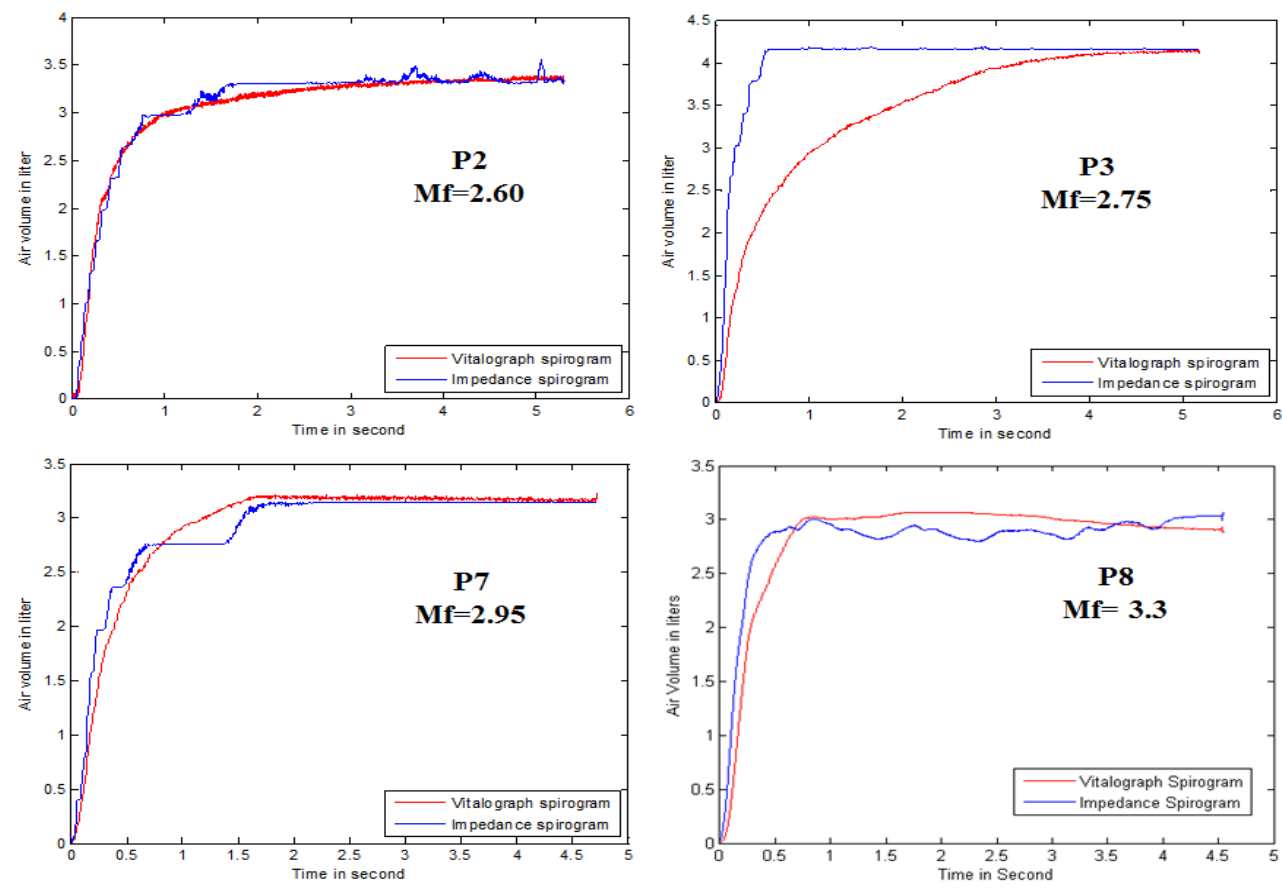

Fig.12: Spirograms - both using Vitalograph and the developed IBS for four subjects. Three were smokers (P2, P7 \& P8) and one had diagnosed asthma (P3). 
it appears that the instrument possibly got saturated, even though the impedance was increasing, there was no change in the output. Thus it could be due to instrumental error and needs to be investigated further.

Correlation between the Vitalograph and IBS curves were obtained for all the eight subjects. Pearson correlation coefficient was calculated using MATLAB function, $\operatorname{corrcoef}(A, B)$, where $A$ stands for a matrix representing air volume measured using Vitalograph and $B$ for impedance measured using IBS. The results are shown in Table 1 which show good correlation for all the 8 subjects, between 0.81 and 0.99 , with a mean of 0.93 and a standard deviation of 0.06 .

Table-1: Analysis for conversion factor, estimated FVC based on the average factor and correlation coefficient

\begin{tabular}{|c|c|c|c|c|c|c|}
\hline Subject & $\begin{array}{c}\text { Transfer } \\
\text { Impedance } \\
\text { Variation } \\
(\Delta \mathrm{z}) \\
\text { ohms }\end{array}$ & $\begin{array}{c}\text { Ventilated } \\
\text { air volume } \\
\text { (Vitalograph) } \\
\text { Litre }\end{array}$ & $\begin{array}{c}\text { Conversion } \\
\text { factor }(\mathrm{CF})\end{array}$ & $\begin{array}{c}\text { Estimated } \\
\text { volume } \\
\text { based on } \\
\text { average } \\
\text { CF } \\
\text { Litre }\end{array}$ & $\begin{array}{c}\text { Error } \\
\%\end{array}$ & $\begin{array}{c}\text { Correlation } \\
\text { Coefficient }\end{array}$ \\
\hline P1 & 1.72 & 4.03 & 2.38 & 4.56 & 13.18 & 0.99 \\
\hline P2 & 1.30 & 3.30 & 2.60 & 3.34 & 1.20 & 0.99 \\
\hline P3 & 1.47 & 4.03 & 2.75 & 3.89 & 3.09 & 0.81 \\
\hline P4 & 1.29 & 3.48 & 2.70 & 3.41 & 1.95 & 0.90 \\
\hline P5 & 1.27 & 2.88 & 2.44 & 3.37 & 17.16 & 0.94 \\
\hline P6 & 1.14 & 2.16 & 2.05 & 3.01 & 39.20 & 0.90 \\
\hline P7 & 1.08 & 3.2 & 2.95 & 2.85 & 10.90 & 0.99 \\
\hline P8 & 0.93 & 3.06 & 3.3 & 2.45 & 19.82 & 0.93 \\
\hline Average & $\mathbf{1 . 2 8}$ & $\mathbf{3 . 2 7}$ & $\mathbf{2 . 6 5}$ & $\mathbf{3 . 3 6}$ & $\mathbf{1 3 . 3 1}$ & $\mathbf{0 . 9 3}$ \\
\hline SD & 0.24 & 0.61 & 0.38 & 0.65 & 12.61 & 0.06 \\
\hline
\end{tabular}

\section{DISCUSSION AND CONCLUSION}

Measurement of forced expiration in spirometry is very useful for diagnosis of several respiratory disorders. In traditional methods a patient has to close the lips of the mouth tightly around a tube with the nose closed using a spring clamp, which is rather uncomfortable and difficult to maneuvers. Besides, the tube itself may pose to be an obstruction, even if it is a little. Furthermore, the traditional types of flow or volume sensors used in spirometers have certain problems; of inertia and friction in case of turbine type sensors, and blocking of mesh grids by droplets of liquids coming out of the mouth when a person breathes into a mesh type sensor. Therefore, an electrical impedance based spirometer that measures the impedance of the thorax with simple clip electrodes attached to hands and legs is attractive from a user point of view. The present work can said to be a preliminary feasibility study for introducing such a simple method in spirometry, particularly in the measurement of forced expiration which includes parameters like Forced Vital Capacity (FVC), and Forced Expiratory Volume (FEV) at certain instants along the procedure.

The instrumentation developed for this preliminary work had some limitations. To get the high sampling frequency needed to acquire $10 \mathrm{kHz}$ signal, the ADC of the PicoScope had to be set for 8 bit resolution. Since the variation in the measured output voltage was only a fraction of the total voltage, the resolution 
was not adequate for a good quality measurement. This was mainly responsible for the large steps, noisy fluctuations and inaccuracies of the spirograms obtained by the IBS. The instrumentation has to be redesigned and improved to avoid these limitations.

Since the thoracic impedance variation was recorded from hands and legs, the movement of the organs of the thorax and the abdomen including the heart also would contribute to the impedance variation along with ventilatory effects. Again, bending of the body during forced expiration may also have an effect. These need to be studied in more detail and ways to exclude such artifacts should be found out, may be using some more electrodes at the mid-thorax region and making multiple impedance measurements using combinations of such electrodes simultaneously.

During measurements, the subjects were instructed to inhale forcefully, hold breath for a while, when they seal the mouth around the tube, then exhale forcefully. However, exhalation by sealing the lips around the air flow tube of the Vitalograph spirometer was difficult. A little air volume may have lost during exhalation which may not contributed to the Vitalograph measurement.

The repeatability of spirograms for a single person was found satisfactory. Correlation between the Vitalograph spirogram, which was taken as the secondary standard in this work, and that obtained using electrical impedance was also reasonably good for all the subjects. Therefore, the electrical impedance technology for an IBS seems to be feasible and needs improvements to eliminate the sources of errors mentioned in this paper.

\section{Acknowledgment}

The authors would like to thank Ministry of Science and Technology, Government of the People's Republic of Bangladesh, and the International Science Programme (ISP) of Uppsala University, Sweden for financial support.

\section{References}

AAMI 1985. American national standard, safe current limits for electromedical apparatus, Association for the Advancement of Medical Instrumentation.

Andreuccetti D, F. R., Petrucci C. 1997. An Internet resource for the calculation of the dielectric properties of body tissues in the frequency range $10 \mathrm{~Hz}-100 \mathrm{GHz}$ [Online]. Available: Website at http://niremf.ifac.cnr.it/tissprop/. IFAC-CNR, Florence (Italy).

Barnes, T. A. \& Fromer, L. 2011. Spirometry use: detection of chronic obstructive pulmonary disease in the primary care setting. Clinical interventions in aging, 6, 47.

Drew, C. \& Hughes, D. 1969. Characteristics of the Vitalograph spirometer. Thorax, 24, 703-706.

Enright, P. L., Johnson, L. R., Connett, J. E., Voelker, H. \& Buist, A. S. 1991. Spirometry in the lung health study: 1. Am Rev Respir Dis, 143, 1215-1223.

Grimnes, S. \& Martinsen, O. 2008. Bioelectricity and bioimpedance basics, Academic Press.

Gupta, A. K. 2011. Respiration rate measurement based on impedance pneumography. Texas Instruments application report SBAA181.

Jat, K. R. 2013. Spirometry in children. Primary Care Respiratory Journal, 22. 
Kadir, M., Baig, T. \& Rabbani, K. 2009. Application of 6-electrode Focused Impedance Method (FIM) to study lungs ventilation. Proc. EIT2009, UK.

Kadir, M. A., Baig, T. N. \& Rabbani, K. S. 2015. Focused impedance method to detect localized lung ventilation disorders in combination with conventional spirometry. Biomedical Engineering: Applications, Basis and Communications, 27, 7.

Kubicek, W., Kinnen, E. \& Edin, A. 1964. Calibration of an impedance pneumograph. Journal of applied physiology, 19, 557-560.

Martinsen, O. G. \& Grimnes, S. 2011. Bioimpedance and bioelectricity basics, Academic press.

Nopp, P., Rapp, E., Pfutzner, H., Nakesch, H. \& Rusham, C. 1993. Dielectric properties of lung tissue as a function of air content. Physics in medicine and biology, 38, 699.

Saxena, N. 2015. Restrictive Lung Disease. The Perioperative Medicine Consult Handbook. Springer.

Schermer, T. R., Verweij, E. H., Cretier, R., Pellegrino, A., Crockett, A. J. \& Poels, P. J. 2012. Accuracy and precision of desktop spirometers in general practices. Respiration, 83, 344-352.

Vijayasekaran, D., Subramanyam, L., Balachandran, A. \& Shivbalan, S. 2003. Spirometry Test. Indian pediatrics, 40, 626-632. 